\title{
Tunable propagation delay of femtosecond pulses in a quantum-dot optical amplifier at room temperature
}

\author{
Poel, Mike van der; Mørk, Jesper; Hvam, Jørn Märcher
}

Published in:

Quantum Electronics and Laser Science Conference, 2005. QELS '05

Link to article, DOI:

10.1109/QELS.2005.1549170

Publication date:

2005

Document Version

Publisher's PDF, also known as Version of record

Link back to DTU Orbit

Citation (APA):

Poel, M. V. D., Mørk, J., \& Hvam, J. M. (2005). Tunable propagation delay of femtosecond pulses in a quantumdot optical amplifier at room temperature. In Quantum Electronics and Laser Science Conference, 2005. QELS '05 (Vol. 3). IEEE. https://doi.org/10.1109/QELS.2005.1549170

\section{General rights}

Copyright and moral rights for the publications made accessible in the public portal are retained by the authors and/or other copyright owners and it is a condition of accessing publications that users recognise and abide by the legal requirements associated with these rights.

- Users may download and print one copy of any publication from the public portal for the purpose of private study or research.

- You may not further distribute the material or use it for any profit-making activity or commercial gain

- You may freely distribute the URL identifying the publication in the public portal 


\title{
Tunable propagation delay of femtosecond pulses in a quantum-dot optical amplifier at room temperature
}

\author{
M. van der Poel, J. Mørk, and J.M. Hvam \\ Research Center COM, Technical University of Demnark, DK-2800 Kgs. Lyngby \\ E-mail: mvp@com.dtu.dk, fax: +45 45936581
}

\begin{abstract}
Optically induced dispersion over a large bandwidth of $2.6 \mathrm{THz}$ is used to slow or speed up a $150 \mathrm{fs}$ pulse in a quantum-dot optical amplifier. A group refractive index change of $4 * 10^{-3}$ is observed.

(C)2000 Optical Society of America

OCIS codes: (250.5980) Semiconductor optical amplifiers; (190.5530) Pulse propagation and solitons
\end{abstract}

\section{Introduction}

The potential of realizing tunable propagation delay of optical pulses using strong, optically induced dispersion in an optical medium has attracted great attention since the pioneering experiments by Hau et al. [1]. Such a tunable propagation delay opens up for optical memory capacity which in turn allows a whole new class of optical components in optical communications systems and signal processing [2]. Recent demonstrations of tunable propagation delay of optical pulses in solid-state optical media [3] at room temperature and in semiconductor quantum wells [4] at cryogenic temperatures suggest that it might be possible to achieve this kind of functionality in practical components.

The above mentioned results showed a large change in group velocity but this was achieved at the cost of a small bandwidth (e.g. $37 \mathrm{~Hz}$ in the case of Bigelow et al. [3] or $2 \mathrm{GHz}$ in the case of $\mathrm{Ku}$ et al. [4]) of the probe signal. In this paper we report measurements of slowing down and speeding up of the propagation of a $150 \mathrm{fs}$ pulse, having a very large bandwidth of $2.6 \mathrm{THz}$, through a quantum-dot (QD) semiconductor amplifier (SOA) at room temperature. This extremely large bandwidth, on the other hand, is at the cost of a rather small group index change of $\Delta \mathrm{n}_{\mathrm{g}}=4 * 10^{-3}$.

\section{Experiment and sample details}

a)

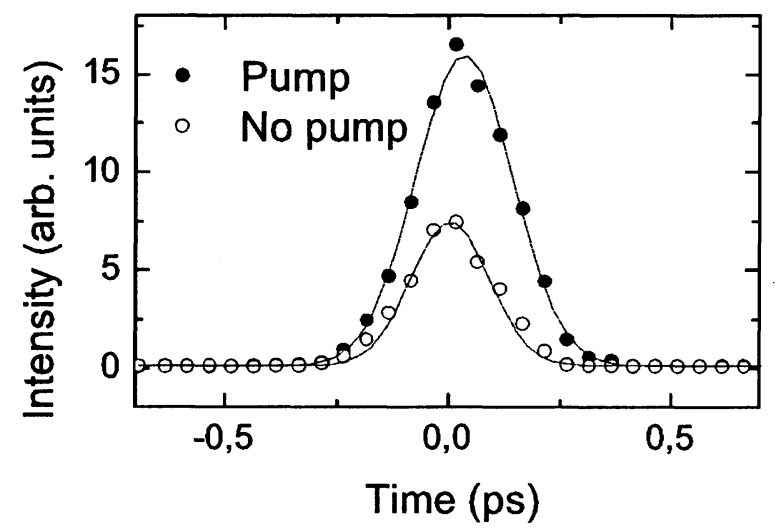

b)

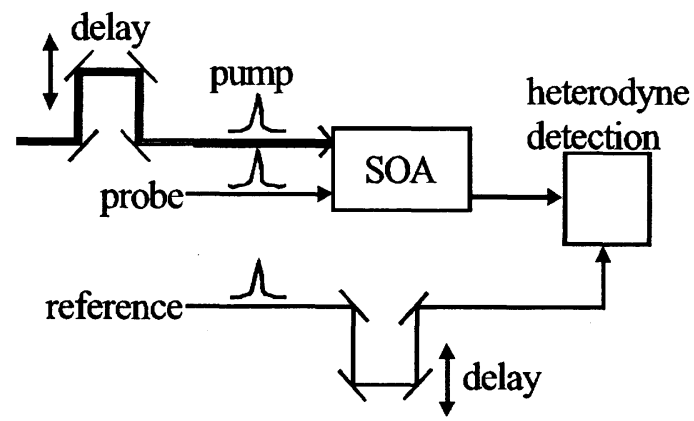

Fig. 1. a) Measurement of pulse delay through cross correlation traces of probe pulse with reference pulse at zero bias current. A 20 fs slow down of the probe pulse is caused by the presence of a pump pulse. Pump bleaching of the absorption also increases the probe transmission. b) Schematic overview of experimental setup.

Cross-correlation traces of a weak probe pulse propagated through a ridge-waveguide QD SOA and a reference pulse were recorded in a heterodyne detection setup described in [5] shown shown schematically in Fig. 1b. Comparison of cross-correlation traces recorded with and without the presence of a strong pump pulse showed changes of probe propagation time, intensity and pulsewidth caused by the pump. An example of this is shown in Fig. 1a. The time shift $\Delta t$, for instance, between arrival time of the probe pulse with and without the presence of the 


\section{JWB96}

pump pulse is related to the pump-induced change in group index $\Delta n_{\mathrm{g}}$ by $\Delta n_{\mathrm{g}}=c \Delta t / L$, where $c$ is the speed of light and $L$ is the length of the component. These pump-induced changes were recorded as function of injected current and pump-probe delay time.

The $2 \mathrm{~mm}$ long and $7 \mu \mathrm{m}$ wide ridge-waveguide SOA had an active layer of 5 sheets of self-organized InAs/InGaAs/GaAs QDs [6] showing a central ground state emission at $1260 \mathrm{~nm}$. The pump, probe and reference pulses were derived from a Ti:Sapphire + regenerative amplifier + optical parametrical amplifier cascade (Coherent Inc.) delivering near-transform limited pulses with $150 \mathrm{fs}$ duration and wavelength tunable in the $950-2000 \mathrm{~nm}$ range. In the experiments reported here, the wavelength was tuned to $1273 \mathrm{~nm}$ - slightly above the GS emission wavelength. The pump intensity is close to the saturation intensity of the amplifier, while the probe intensity, being about two orders of magnitude smaller, is in the small-signal regime.

\section{Results and discussion}

The pump-induced change in probe travel time for different bias currents at zero pump-probe delay is shown in Fig. 2. For the zero injected current, a pump-induced delay of the probe of $17 \mathrm{fs}$ is seen. As bias current is increased the slowdown diminishes, changes sign, and becomes a speeding up of $10 \mathrm{fs}$ at high injected current. We qualitatively interpret this behavior in the following way: At zero bias the pump pulse injects a population of carriers into the QD ground state. These carriers result in a spectral gain and index feature centered on the pump wavelength resulting in a decreased probe group velocity. At high injection current the pump pulse burns a spectral hole creating a spectral feature of similar nature as for zero bias current, but of opposite sign resulting in an increased probe group velocity. In order to understand this quantitatively we write the optical susceptibility seen by the probe, $\chi$, as

$$
\chi(\omega)=\int_{0}^{\infty} d \omega^{\prime} \frac{N e^{2}|D|^{2}}{3 \varepsilon_{0} \square} \frac{\omega^{\prime}-\omega+i \gamma^{\prime}}{\left(\omega^{\prime}-\omega\right)^{2}+\gamma^{\prime 2}\left(1+s_{0}\right)}+\chi_{b g}
$$

where $N=\left(N_{\mathrm{gg}}-N_{\mathrm{ex}}\right)$ is the difference in density of ground and excited state dots, $e$ is the fundamental charge, $D$ is the $\mathrm{QD}$ dipole moment, $\varepsilon_{0}$ is the vacuum permittivity, $\square$ is Planck's constant, $\square \gamma^{\prime}$ is the homogeneous QD linewidth, $s_{0}$ is an intensity saturation parameter, and $\chi_{\text {bg }}$ is the background susceptibility. Note that the inhomogeneous QD broadening and the laser linewidth are included in Eq. 1 through the frequency dependence of $N$ and $s_{0}$. Given the susceptibility, the group index $n_{\mathrm{g}}$ can be calculated as

$$
n_{g}=\sqrt{1+\chi}+\omega \frac{d}{d \omega} \sqrt{1+\chi}
$$

For the case of zero injection current, and using realistic parameters for our SOA, Eq. 1 gives a maximum slowdown of $31 \mathrm{fs}$, in reasonably good agreement with experiment. Furthermore, Eq. 1 predicts that the pump-induced change of the probe travel time through the amplifier is proportional to the dot inversion. The dot inversion itself is proportional to the logarithm of the on-chip gain. In Fig. 2 this proportionality of delay time and logarithm of onchip gain is clearly demonstrated.

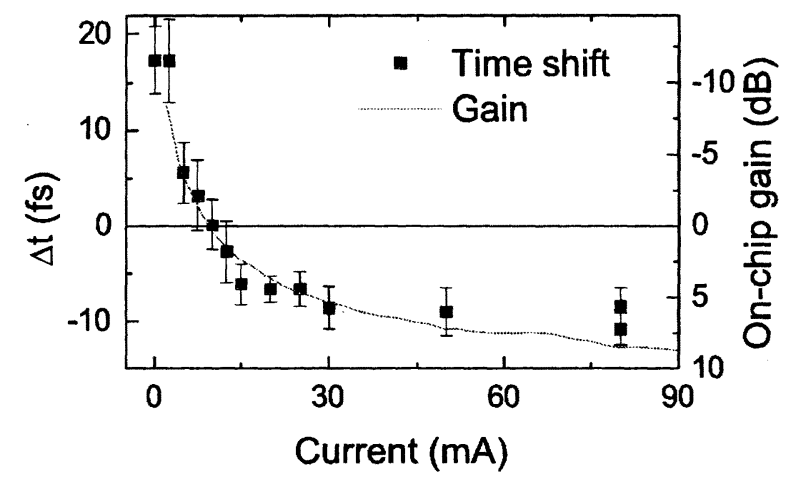

Fig. 2. Left axis: pump-induced change in probe travel time as function of injection current. Right axis: Independently measured small signal gain of the amplifier. 


\section{JWB96}

The pump injected carriers at zero bias current have a lifetime of the order of $100 \mathrm{ps}$. At the same time, the amplified spontaneous emission from the amplifier shows a separation of $60 \mathrm{meV}$ between the ground-state and the excited-state, several times larger than the room temperature thermal energy. Together, the long lifetime and large dot level separation imply the persistence of the pump-induced susceptibility long after passage of the probe pulse. Fig. 3 shows the pump-induced change in probe travel time at zero bias current as function of pump-probe delay time. The decay in probe delay time matches well the decay in the pump-induced difference in probe transmission also shown in Fig. 3. Again, the differential transmission reflects the change in pump-induced dot inversion. This behavior is therefore in agreement with the expectations from Eq. 1. The slightly faster decay of the probe slowdown compared to the differential probe transmission seen in Fig. 3 suggests some degree of spreading of the pump-injected carriers. In the gain region of the SOA, the probe speed up does not persist to long pump-probe delays. This is due to the fast, picosecond recovery of the pump-induced spectral feature which is possible through intra-dot relaxation.

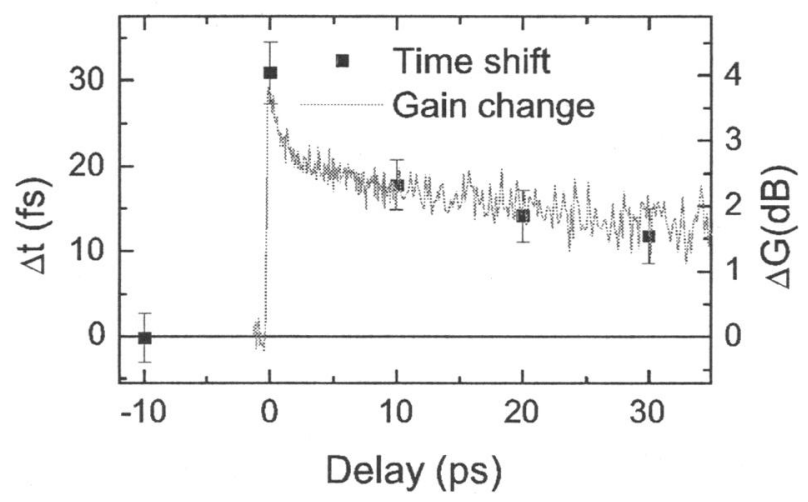

Fig. 3. Left scale: pump-induced change in probe travel time as function of pump-probe delay. Right scale: pump-induced change in probe transmission.

\section{Conclusion}

We have demonstrated optical tuneability of the travel time of a probe pulse propagating trough a QD SOA. The overall change in travel time was $30 \mathrm{fs}$ corresponding to a group velocity index change of $\Delta n_{\mathrm{g}}=4^{*} 10^{-3}$. The bandwidth over which this delay occurs is $2.6 \mathrm{THz}$.

The results are interpreted in a simple model of the pump-induced changes in optical susceptibility, which explains well the observations. The mechanism behind the slowing down and speeding up of the pulse is related to, but no similar to the effect of coherent population oscillations described in litterature [3] in the sense that it relies on the carrier lifetime in the excited state but is relatively insensitive to quantum decoherence of those carriers.

\section{Acknowledgements}

Alexey Kovsh of the Ioffe Institute is acknowledged for growing the QD sample. Matthias Laemmlin and Dieter Bimberg of Technische Universität Berlin is acknowledged for wafer processing. This project was supported by the EU-IST project DOTCOM.

\section{References}

[1] L.V. Hau, S.E. Harris, Z. Dutton, and C.H. Behroozi, "Light speed reduction to 17 meters per second un an ultracold atomic gas", Nature $397,594-598(1999)$

[2] C.J. Chang-Hasnain, P.-C. Ku, J. Kim, S.-L. Chuang, "Variable optical buffer using slow light in semiconductor nanostructures", Proc. IEEE 91, $1884-1897(2003)$

[3] M.S. Bigelow, N.N. Lepeshkin, and R. Boyd, "Observation of ultraslow light propagation in a ruby crystal at room temperature", Phys. Rev. Lett. 90, 113903-1-4 (2003)

[4] P.-C. Ku, F. Sedgwick, C.J. Chang-Hasnain, P. Palinginis, T. Li, H. Wang, S.-W. Chang, and S.-L. Chuang, "Slow light in semiconductor quantum wells", Opt. Lett. 19, $2291-2293$ (2004)

[5] P. Borri, W. Langbein, J. Mørk, and J.M. Hvam, "Heterodyne pump-probe and four-wave mixing in semiconductor optical amplifiers using balanced lock-in detection", Opt. Comm. 169, 317-324 (1999)

[6] A.R. Kovsh et al., "InAs/InGaAs/GaAs quantum dot lasers of $1.3 \mathrm{~mm}$ range with enhanced optical gain", J. Crystal Growth 250, 729 (2003) 